\title{
FREQUENCY OF GENERALIZED ANXIETY DISORDERS IN PATIENTS WITH POLYCYSTIC OVARIAN SYNDROME
}

\author{
Rabia Asghar, Usama Bin Zubair*, Syed Azhar Ali**, Ayaz Muhammad Khan ${ }^{* * *}$, Aneel Shafi ${ }^{* * * *}$, Hafiz Shafique Ahmad ${ }^{* * * *}$ \\ PAF Hospital Lahore Cantt Lahore Pakistan, *Mater Misericordiae University Hospital Dublin, ${ }^{* *}$ Combine Militry Hospital Rawalakot Cantt, Rawalakot AJK \\ National University of Medical Sciences (NUMS) Pakistan, ${ }^{* * *}$ Fatima Memorial Medical Collage Lahore, ${ }^{* * * *}$ Avicenna Medical Collage Lahore,

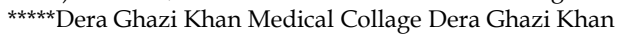

\begin{abstract}
Objective: To determine the factors associated with generalized anxiety disorders in patients presenting with polycystic ovarian syndrome.

Study Design: Cross-sectional study.

Place and Duration of Study: Gynecology and Psychiatric outpatient department of Pakistan Institute of Medical Sciences, SZABMU, Islamabad, from Jan to Jul 2016.

Methodology: Patients presenting with polycystic ovarian syndrome were enrolled. Patients were selected from the gynaecological and Medicine department. Patient's history was taken and diagnosis of generalized anxiety disorder was made utilising Generalized Anxiety Disorder-7. Patients having diabetes mellitus, hypertension, Cushing's syndrome, hypoglycaemia, co-morbid psychiatric illnesses, history of psychiatric illness in past and family history of psychiatric illness were excluded from the study.

Results: Among 105 patients with polycystic ovarian syndrome, 79 (75\%) patients had no generalized anxiety disorders while, $26(25 \%)$ had generalized anxiety disorders. 2 out of $12(16.6 \%)$ patients of age range of 15-20 year had generalized anxiety disorders, 9 out of $6314.2 \%$ patients having age 21-30 years had generalized anxiety disorders and 15 out of 30 (50\%) patients having age 31-40 years age had generalized anxiety disorders. Chi-square test revealed age, education level and socioeconomic status has a significant association with presence of generalized anxiety disorder among patients of polycystic ovarian disorder (with $p$-value <0.05).

Conclusion: Generalized anxiety disorder was common in patients with polycystic ovarian syndrome. Age, low education level and low socioeconomic status had a significant relationship with generalized anxiety disorder in polycystic ovarian syndrome patients.
\end{abstract}

Keywords: Generalized anxiety disorder, Polycystic ovarian syndrome, Psychiatric illness.

\footnotetext{
This is an Open Access article distributed under the terms of the Creative Commons Attribution License (https://creativecommons.org/licenses/by-nc/4.0/), which permits unrestricted use, distribution, and reproduction in any medium, provided the original work is properly cited.
}

\section{INTRODUCTION}

Polycystic ovary syndrome is an endocrine disorder that occurs commonly and is associated with various abnormalities in psychological domain in addition to reproductive and metabolic problems. Its main features include abnormalities and irregularities in menstrual cycle, abnormally high levels of androgens, hirsutism, infertility and ultrasound appearance of polycystic ovaries ${ }^{1}$. International data on prevalence of PCOD shows high degree of variability with ranges from $2.2-26 \%$. Community studies that were done in Chinese and Sri Lankan communities, utilizing Rotterdam's criteria show prevalence of $2-7 \%$ and $6.3 \%$ respectively. Studies on Caucasian populations show a prevalence of PCOS 5-8\% and in Australian women prevalence is of $11.9 \%^{2}$.

Generalized anxiety disorder (GAD) is characterized by "extreme worrying about work, social relationships, or financial matters almost every day for six

Correspondence: Dr Rabia Asghar, Consultant Psychiatrist, Pakistan Air Force Hospital, Lahore Pakistan

Received: 26 Jun 2019; revised received: 02 Jan 2020; accepted: 08 Jan 2020 months or more." A little work has been done in relation to the symptoms of anxiety in polycystic ovary syndrome, although generalized anxiety disorder is most common psychiatric disorder among endocrine patients especially in polycystic ovary syndrome. Since the prevalence is high, and condition is easily manageable yet very disabling and distressing, clinicians should pay more attention to the association of generalized anxiety disorder and $\mathrm{PCOS}^{3}$. The co-morbidities in PCOS include high blood pressure, lipid abnormalities, diabetes and generalized anxiety disorders ${ }^{4}$.

A study by Dokras indicated increased anxiety symptoms in women with PCOS. The prevalence of generalized anxiety symptoms was available in four studies and was significantly higher in PCOS subjects $(42 / 206,20.4 \%)$ than that in controls $(8 / 204,3.9 \%)^{5}$. PCOS has high prevalence of psychiatric disorders with $52.7 \%$, suffering from at least one mental health problem. Among them, 23\% suffered from major depressive disorder (compared to $7.5 \%$ of controls), $1.8 \%$ from dysthymic disorder, $15.45 \%$ from panic disorder (compared to $5 \%$ of controls), $6.36 \%$ had obsessive 
compulsive disorder (compared to $2.5 \%$ of controls), $8 \%$ cases harboured suicidal tendency while rates for bipolar disorder and GAD were $2.72 \%$ and $15.45 \%$ respectively. These figures imply that we should give attention towards screening these patients for psychiatric disorders and help them in getting timely intervention ${ }^{1}$.

This study was conducted with a view to take a proactive approach to give awareness to the people of community about the co-morbidity of polycystic ovary syndrome and generalized anxiety disorder. In co-existence of generalized anxiety disorder, the treatment of polycystic ovary syndrome becomes more difficult as anxiety further aggravates menstrual irregularities. Secondly limited local data available about the prevalence of generalized anxiety disorder (GAD) in polycystic ovary syndrome (PCOS) so the study will help to build up local data.

\section{METHODOLOGY}

The cross-sectional study study was conducted in Psychiatry and Gynaecology OPD of Pakistan Institute of Medical Sciences (PIMS), SZABMU Islamabad, from January to July 2016. Sample size was calculated with WHO sample size calculator according to following assumptions: population proportion $=15.4 \%^{7}$, confidence level $=95 \%$ and Absolute precision $=7 \%$. Sample size turned out to be 105 patients. Non-probability consecutive sampling technique was used to gather the data. The female patients having age 15-40 years regardless of marriage status, diagnosed cases of polycystic ovary syndrome, presenting with either of the two features like menstrual irregularities, hirsutism and anovulation for $>6$ weeks were included in study. Patients with DM, HTN, Cushing's syndrome, already diagnosed psychiatric illness, past or family history of psychiatric illness were excluded from our study.

Generalized Anxiety Disorder was characterized by "extreme worrying about work, social relationships, or financial matters almost every day for six months or more", diagnosed by using GAD-7 Score. Score $>8$ for $>2$ weeks was diagnostic of GAD.

Polycystic ovarian syndrome; rotterdam criteria: PCOS to be present if any 2 out of 3 criteria are met. Oligo-ovulation and/or anovulation, was diagnosed by taking history from patients (like irregular menses or absent menstrual cycles). Excess androgen activity as evidenced by acne, excessive hair growth on face and body, by taking history from patients. Polycystic ovaries ( $\geq 12$ Antral Follicles in one ovary or ovarian volume $\left.\geq 10 \mathrm{~cm}^{3}\right)$, were diagnosed by pelvic ultrasonography.

All the patients' already diagnosed as case of polycystic ovarian syndrome were included in the study. Permission was taken from the hospital ethics committee. Sample was collected by non-probability consecutive sampling technique. Informed written consent was taken from the study subjects. History including age, gender, weight and height was taken at the start of study from each patient included in the analysis. Examination was done with special emphasis on signs and symptoms of polycystic ovary syndrome; Cushing's syndrome was verified by a senior colleague (Registrar Medicine). Then questions were asked from the patients about generalized anxiety disorders according to GAD-7. A complete ultrasonographic examination was done by consultant radiologist. The report was prepared by consultant radiologist and was collected by post graduate trainee. Then blood was drawn in serum bottle to look for fasting blood sugar after an overnight fast (8-to 12-hour fast) to exclude diabetes mellitus and hypoglycemia. Then patients were asked to collect urine sample in sterile urine R/E bottle that was sent to PIMS laboratory for checking the sugars in urine. All other tests were done from the hospital laboratory. The information collected was then transferred to the prescribed proforma.

We analysed data using statistical package for social sciences version (SPSS-23). Variables like age, education status, marital status, number of children, socioeconomic status, family history of psychiatric illness and past history of psychiatric illness, were noted from the proformas. Quantitative variables including age and BMI were presented by mean \pm SD. Frequencies and percentages were calculated for qualitative variables i.e. generalized anxiety disorders. We used stratification in order to control effect modifiers such as age. Chi square test was applied. $p$-value $\leq 0.05$ was considered significant in our study.

\section{RESULTS}

There were total 105 patients included in the study. The mean age of the patients in the study was $28.10 \pm 12.46$ years [range 15-40 years] as in table-I. Our study showed that $12(11.5 \%)$ of patients had their education up to primary level, 19 (18\%) of patients had their education up to middle class, 38 (36\%) of patients had their education up to matriculation, 24 (22.9\%) of patients had their education up to intermediate level and $12(11.50 \%)$ of patients had their education up to post graduate level. In our study, $66(63 \%)$ patients 
were married while $39(37 \%)$ patients were unmarried. The mean weight of study patients was $63.5 \pm 5.8$ kilograms ranging from 52-76. The mean height was $169.5 \pm 13 \mathrm{~cm}$ with a range of $15.3-179.6 \mathrm{~cm}$. The BMI of study patients were $21.8 \pm 1.3$ ranging between 19-24.7. Among 105 patients with PCOs, 79 (75\%) patients had no generalized anxiety disorders while $26(25 \%)$ had generalized anxiety disorders (figure). Table-II shows significant association i.e, $p<0.01$ with presence of GAD among patients of PCOS for demographic variables.

Table-I: Sociodemographic profile of patients with polycystic ovarian syndrome (pcos) having generalized anxiety

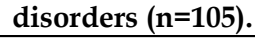

\begin{tabular}{l|c}
\hline Variables & No. of Patients (\%) \\
\hline Age (Years) & $12(11.4)$ \\
\hline $15-20$ & $63(60)$ \\
$31-30$ & $30(28.6)$ \\
\hline Educational Level \\
\hline Primary & $12(11.4)$ \\
Middle & $19(18.1)$ \\
Matriculation & $38(36.2)$ \\
Intermediate & $24(22.9)$ \\
Post-graduation & $12(11.4)$ \\
\hline Socioeconomic Status & $32(30.5)$ \\
\hline Poor & $27(25.7)$ \\
Middle Class & $46(43.8)$ \\
High Class & $66(62.9)$ \\
\hline Marital Status & $39(37.1)$ \\
\hline Married &
\end{tabular}

Table-I: Association of socio-demographic profile of patients with polycystic ovarian syndrome (PCOs) with generalized anxiety disorders $(n=105)$.

\begin{tabular}{|c|c|c|c|c|}
\hline \multirow[t]{2}{*}{ Variables } & \multirow{2}{*}{$\begin{array}{c}\text { No. of } \\
\text { Patients }\end{array}$} & \multicolumn{2}{|c|}{$\begin{array}{c}\text { Generalized } \\
\text { Anxiety Disorder }\end{array}$} & \multirow{2}{*}{$\begin{array}{c}p \text { - } \\
\text { value }\end{array}$} \\
\hline & & Yes & No & \\
\hline \multicolumn{5}{|l|}{ Age (years) } \\
\hline $15-20$ & 12 & $2(16.6)$ & $10(83.4)$ & \multirow{3}{*}{$<0.01$} \\
\hline $21-30$ & 63 & $9(14.2)$ & $54(85.8)$ & \\
\hline $31-40$ & 30 & $15(50)$ & $15(50)$ & \\
\hline \multicolumn{5}{|c|}{ Educational Level } \\
\hline Primary & 12 & $2(16.6)$ & $10(83.4)$ & \multirow{6}{*}{$<0.01$} \\
\hline Middle & 19 & $3(15.7)$ & $16(84.3)$ & \\
\hline Matriculation & 38 & $10(26.3)$ & $28(73.7)$ & \\
\hline Intermediate & 24 & $7(29.1)$ & $17(70.9)$ & \\
\hline Post- & & & & \\
\hline Graduation & 12 & $4(33.3)$ & $8(66.7)$ & \\
\hline \multicolumn{5}{|c|}{ Socioeconomic Status } \\
\hline Poor & 32 & 05 (15.6) & $27(84.4)$ & \multirow{3}{*}{$<0.01$} \\
\hline Middle Class & 27 & $3(11.1)$ & $24(88.9)$ & \\
\hline High Class & 46 & $18(39.1)$ & $28(60.9)$ & \\
\hline \multicolumn{5}{|l|}{ Marital Status } \\
\hline Married & 66 & $15(22.7)$ & $51(77.3)$ & \multirow{2}{*}{$<0.01$} \\
\hline Unmarried & 39 & $11(28.2)$ & $28(71.8)$ & \\
\hline
\end{tabular}

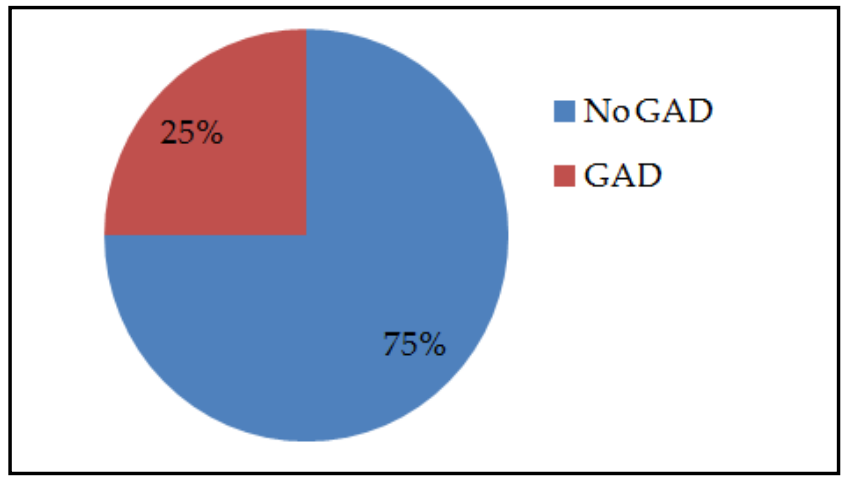

Figure: Distribution of polycystic ovarian syndrome's patients having generalized anxiety disorders $(n=105)$.

\section{DISCUSSION}

Women having polycystic ovary syndrome (PCOS) have gynaecologic, medical and psychological problems in their life. Recent studies showed an elevated risk of anxiety and mood disorders in such women. Annagür et al , did study between March 2011 and February 2012 comprising of 73 in three groups. PCOS patients with only major depressive disorder $(n=23)$, PCOS patients with only generalized anxiety disorder $(n=20)$, and PCOS patients without any diagnosed psychiatric condition (not diagnosed-ND group, $n=30$ ). It was found that 17-OHP levels were significantly raised in the MDD group than in the ND group. DHEAS levels were considerably greater in the MDD group and the GAD group than in the ND group ${ }^{5}$.

A study by Mujtaba et $a l^{6}$, indicated that the onethird of PCOS patients (33\%) suffered from anxiety symptoms. Mansson et $a l^{7}$, also found that both social phobia (27\%) and GAD (13\%) have a higher incidence in such patients. There is enough data to support the concept that anxiety is a risk factor for the development of depressive disorders. When compared with our study the prevalence of generalized anxiety disorders was $26 \%$ similar to study findings by Battachariya et $a l^{8}$. Our study didn't find any significant association of demographic factors with GAD incidence in PCOS patients. In a systematic review of 12 comparative studies Barry et $a l^{9}$, reported higher prevalence of depression $(Z=17.92, p<0.001$; Hedges' $\mathrm{g}=0.82$; $95 \%$ CI 0.73 $0.92)$ and anxiety $(Z=5.03, p<0.001$; Hedges' $g=0.54 ; 95 \%$ CI 0.33-0.75) scores in the participants with, than without, PCOS. Women with PCOS with lower BMI had lower scores in anxiety and depression. However our study showed that higher BMI increases the prevalence of generalized anxiety disorder and vice versa. Women with poor background 5 out of $32(15.6 \%)$ and high class families 18 out of $46(39.1 \%)$ had more GAD than 
women with middle class families. However there was no significant difference between married, ( 15 out of 66 $(22.7 \%)$ women had generalized anxiety disorders) and unmarried 11 out of 39 (28.2\%) women had generalized anxiety disorders. Among 105 patients with PCOs, 17 out of $31(55 \%)$ patients having family history of Psychiatric illness had generalized anxiety disorders, however, 9 out of 74 (12\%) having no family history of Psychiatric illness had generalized anxiety disorders. Among 105 patients with PCOs, 19 out of 21 (90.4\%) patients having past history of Psychiatric illness had generalized anxiety disorders, however, 7 out of 84 $(8.3 \%)$ having no past history of Psychiatric illness had generalized anxiety disorders.

Out of $50 \%$ patients had at least one co-morbid psychiatric disorder. Major depression (33\%) was most common and generalized anxiety disorder was next most common disorder in the list (13.6\%) and bingeeating disorder $(6.8 \%)^{10}$. When compared with our study the prevalence of generalized anxiety disorders was $25 \%$ and it is more common in patients having past history of psychiatric illness $90 \%$ or having family psychiatric illness 55\%. Comparison between psychological well-being in women suffering from PCOS showed no significant difference in socio-demographic parameters; therefore, age, marital status, education had no influence on the prevalence of anxiety in women with PCOS or their mental health status. History of no significant difference in body mass index and insulin resistance index scores was found between women with and without psychiatric disorders $(p>0.05$ for both comparisons). Our study showed that higher BMI increases the prevalence of generalized anxiety disorders and vice versa. A considerable number of women with PCOS experience a psychiatric disorder during their lifetime. Clinicians should be aware that women with PCOS are at a high risk for major depression, generalized anxiety disorder, and bingeeating disorder.

Arshad et al, enrolled 110 patients of PCOS and evaluated them using diagnostic and statistical manual for mental disorders, fourth edition criteria by means of mini international neuropsychiatric interview, English version 5.0.0. Diagnosis of PCOS was made according to the National Institute of Health/National Institute of Child Health and Human Development, 1990 consensus conference criteria. Forty subjects without PCOS who were matched for age and body mass index were taken as a comparison group. About $23 \%$ of cases had major depressive disorder as compared to $7.5 \%$ of controls, $1.8 \%$ had dysthymic disorder, $15.45 \%$ had panic disorder compared to 5\% of controls, $6.36 \%$ had obsessive compulsive disorder compared to $2.5 \%$ of controls, $8 \%$ cases had suicidal tendencies, $2.72 \%$ of cases were bipolar affective disorder, and $15.45 \%$. had generalized anxiety disorder (GAD) ${ }^{11-14}$. When compared with our study we included 105 patient in our study slightly lower in number, the prevalence of generalized anxiety disorders was $26 \%$ and it is more common in patients having past history of psychiatric illness $90 \%$ or having family history of psychiatric illness 55\%. Comparison between psychological wellbeing in women suffering from PCOS showed no significant difference in socio-demographic parameters; therefore, age, marital status, education had no influence on the prevalence of anxiety in women with PCOS or their mental health status. No significant difference in body mass index and insulin resistance index scores was found between women with and without psychiatric disorders ( $p>0.05$ for both comparisons).

In a study on gynaecologist referred PCOS patients in Taiwan, Jeng-Hsiu et al ${ }^{12}$, identified anxiety disorder (HR 1.392) and sleep disorder (HR 1.495) were more prevalent among the PCOS patients than among the patients in the comparison cohort. Other studies also supported this fact and results were in accordance with our study ${ }^{16-18}$.

\section{CONCLUSION}

Generalized anxiety disorder was found common in patients with polycystic ovarian syndrome. Increased age, lower education level and low socioeconomic status had a significant relationship with GAD in PCOS patients.

\section{CONFLICT OF INTEREST}

This study has no conflict of interest to be declared by any author.

\section{REFERENCES}

1. Hussain A, Chandel RK, Ganie MA, Dar MA, Rather YH, Wani ZA, et al. Prevalence of psychiatric disorders in patients with a diagnosis of polycystic ovary syndrome in kashmir. Ind J Psychol Med 2015; 37(1): 66-70.

2. Joshi B, Mukherjee S, Patil A, Purandare A, Chauhan S, Vaidya R. A cross-sectional study of polycystic ovarian syndrome among adolescent and young girls in Mumbai, India. Ind J Endocrinol Metab 2014; 18(3): 317-24.

3. Antony AC, Berry RJ, Cherian A, Stabler SP. Psychiatric disorders (generalized anxiety disorders). Goldman L, Ausiello D. Cecil Text Book of Medicine. 25 th ed. New Dehli: Elsevier 2014; 34(3): 1270-80.

4. Sirmans SM. Epidemiology, diagnosis, and management of polycystic ovary syndrome. Clin Epidemiol 2013; 6(1): 1-13.

5. Dokras A, Clifton S, Futterweit W, Wild R. Increased prevalence of anxiety symptoms in women with polycystic ovary syndrome: 
systematic review and meta-analysis. Fertil Steril 2012; 97(1): 225-30.

6. Annagür BB, Tazegül A, Uguz F, Kerimoglu ÖS, Tekinarslan E, Celik Ç. Biological correlates of major depression and generalized anxiety disorder in women with polycystic ovary syndrome. J Psychosom Res 2013; 74(3): 244-47.

7. Johnson PL, Truitt W, Fitz SD, Minick PE, Dietrich A, Sanghani S, et al. A key role for orexin in panic anxiety. Nat Med 2010; 16(1): 111-15.

8. Weiner CL, Primeau M, Ehrmann DA. Androgens and mood dysfunction in women: comparison of women with polycystic ovarian syndrome to healthy controls. Psych Med 2004; 66(3): 356-62.

9. Greist JH, Jefferson JW, Kobak KA, Katzelnick DJ, Serlin RC. Efficacy and tolerability of serotonin transport inhibitors in obsessive-compulsive disorder. A meta-analysis. Arch Gen Psych 1995; 52(1): 53-60.

10. Barry JA, Kuczmierczyk AR, Hardiman PJ. Anxiety and depression in polycystic ovary syndrome: a systematic review and meta-analysis. Hum Reprod 2011; 26(9): 2442-51.

11. Annagür BB, Kerimoglu ÖS, Tazegül A, Gündüz Ş, Gençoglu BB. Psychiatric comorbidity in women with polycystic ovary syndrome. J Obstet Gynaecol Res 2015; 41(8): 1229-33.

12. Hussain A, Chandel RK, Ganie MA, Dar MA, Rather YH, Wani
ZA, et al. Prevalence of psychiatric disorders in patients with a diagnosis of polycystic ovary syndrome in kashmir. Ind J Psychol Med 2015; 37(1): 66-70.

13. Hung JH, Hu LY, Tsai SJ, Yang AC, Huang MW, Chen PM, et al. Risk of psychiatric disorders following polycystic ovary syndrome: a nationwide population-based cohort study. PLoS One 2014; 9(5): e97041-45.

14. Rassi A, Veras AB, dos Reis M, Pastore DL, Bruno LM, Bruno $\mathrm{RV}$, et al. Prevalence of psychiatric disorders in patients with polycystic ovary syndrome. Compr Psych 2010; 51(6): 599-602.

15. Hollinrake E, Abreu A, Maifeld M, Van Voorhis BJ, Dokras A. Increased risk of depressive disorders in women with polycystic ovary syndrome. Fertil Steril 2007; 87(6): 1369-76.

16. Kerchner A, Lester W, Stuart SP, Dokras A. Risk of depression and other mental health disorders in women with polycystic ovary syndrome: a longitudinal study. Fertil Steril 2009; 91(1): 207-12.

17. Rodgers RJ, Avery JC, Moore VM, Davies MJ, Azziz R, StenerVictorin E, et al. Complex diseases and co-morbidities: polycystic ovary syndrome and type 2 diabetes mellitus. Endocr Connect 2019; 8(3): R71-R75.

18. Chaudhari AP, Mazumdar K, Mehta PD. Anxiety, depression, and quality of life in womenwith polycystic ovarian syndrome. Ind J Psychol Med 2018; 40(3): 239-46. 